\title{
Extended time series of Solar Activity Indices (ESAI): new possibilities for complex description of magnetic cycle
}

\author{
Yu.A. Nagovitsyn ${ }^{1}$, V.G. Ivanov ${ }^{1}$, E.V. Miletsky ${ }^{1}$ \\ and D.M. Volobuev ${ }^{1}$ \\ ${ }^{1}$ Central astronomical observatory at Pulkovo of RAS, St.-Petersburg, Russia \\ email: nag@gao.spb.ru
}

\begin{abstract}
A new information resource to study solar magnetic field variations and their influence on the Earth extending ordinary lengths of some traditional indices of solar activity is introduced.
\end{abstract}

\section{Introduction}

One of the "hottest" areas in modern scientific researches is the study of long-term climatic changes. In framework of this problem the investigations of solar activity variations in the past are urgent owing to the solar-terrestrial climate links. However, we lack sufficient regular and complex observational data for "history of the Sun" even during relatively short (centennial and multi-centennial) time scales let alone information through long-term (millennial or multi-millennial) period. This paper is devoted to multi-centennial time interval mainly. The primary goal here is the most complex description of the solar magnetic field cyclic variations, parametrized by different indices. More long time scales in other papers of our team in this book are examined (Miletsky et al.; Volobuev et al.).

\section{Methods and results}

The database hereinafter referred to as ESAI (Extended time series of Solar Activity Indices) is a new information resource located at http://www.gao.spb.ru/database/esai. ESAI includes original observational, synthetic and simulated sets to study long-term solar magnetic field variations and their influence on the terrestrial processes. ESAI extends ordinary lengths of some traditional indices of solar activity: sunspot areas, Wolf numbers (i.e. equatorial component of the solar global magnetic field), polar faculae numbers (polar component of the field), sunspot mean latitudes and north-south asymmetry of hemispheres (N-S location of activity).

The monthly sunspot areas (1821-1989), the yearly sunspot areas (1821-1994) and the yearly mean latitudes of sunspots (1854-1985) were created by compilation of preGreenwich observational data sets (by Schwabe, Carrington, De La Rue, Sporer) and post-Greenwich observations (by Gnevysheva) to the Greenwich general system. The yearly polar faculae numbers were constructed by synthesis of different data series: Mt.Wilson, Greenwich, Lyon, Kodaykanal, Tokyo, Zurich, Kislovodsk observations of polar faculae and observations of polar coronal structures during solar eclipses. The series of yearly Wolf numbers for 1090-2003 were reconstructed using the Krylov-Bogolyubov approach to description of weakly nonlinear oscillatory processes, on the one hand, and 

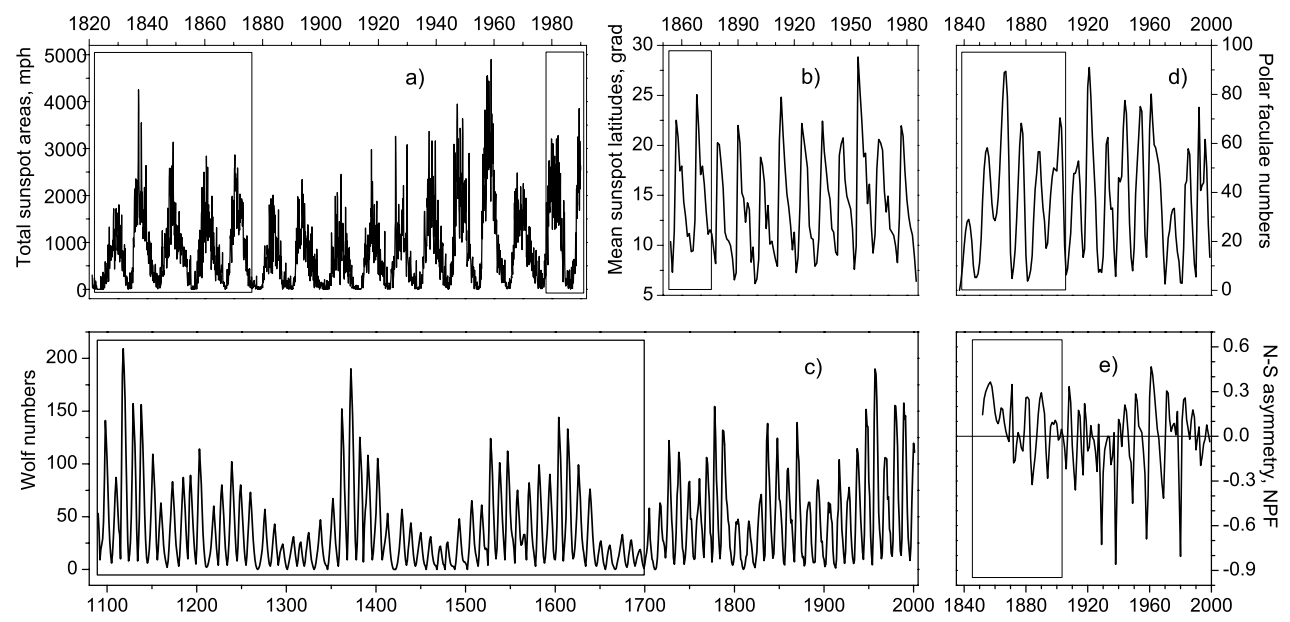

Figure 1. Some time series from ESAI database: a) the observational monthly total sunspot areas, b) the yearly mean latitudes of sunspots; c) simulated series of yearly Wolf numbers; d) synthetic series of yearly means of polar faculae numbers, e) N-S asymmetry of polar faculae numbers. The ESAI-extensions of series are marked as the rectangular borders.

Series

- monthly sunspot areas (Greenwich general system)

- yearly sunspot areas (N- and S-hemispheres)

- yearly mean latitudes of sunspots for N- and S-

hemispheres

- yearly polar faculae numbers (Mt.Wilson general system) for N- and S- hemispheres

- yearly Wolf numbers (Zurich general system)

- yearly polar faculae numbers (Mt Wilson general system)

$\begin{array}{ccc}\text { Conventional, } T_{C} & E S A I, T_{E} & T_{E} / T_{C} \\ 1874-1976 & 1821-1989 & 1.64 \\ 1874-1976 & 1821-1994 & 1.70 \\ & & \\ 1874-1976 & 1854-1985 & 1.28 \\ & & \\ 1906-1991 & 1837-1999 & 1.88 \\ 1700-2003 & 1090-2003 & 3.02 \\ & & \\ 1906-1991 & 1705-1999 & 3.42\end{array}$

Table 1. Comparison of conventional and ESAI time intervals: information gain $T_{E} / T_{C}$

the well-known Schove data on the epochs of extrema of 11-yr solar cycles in the past, on the other. The yearly polar faculae numbers for 1705-1999 were created by analogous procedure (in assumption that 11-yr cycles of polar faculae develop in exact antiphase to sunspot cycles).

Necessary references to our publications as well as full text of presentation of ESAI database can be found at the above-mentioned Internet address.

\section{Acknowledgements}

The work was supported by grants: INTAS No 2000-0752 and 2001-0550, FSTP "Astronomy" No 1105, Programs of Presidium of RAS "Non-stationary phenomena in astronomy" and OPhN-16, RFBR No 01-07-90289, 03-02-17505, 04-02-17560. 